\title{
CORRECTION
}

View Article Online

View Journal I View Issue

W) Check for updates

Cite this: J. Mater. Chem. A, 2022, 10 337

DOI: $10.1039 / d 1$ ta90264d

rsc.li/materials-a

\section{Correction: Nitride MXenes as sulfur hosts for thermodynamic and kinetic suppression of polysulfide shuttling: a computational study}

Ke Fan, ${ }^{a}$ Yiran Ying, ${ }^{a}$ Xin Luo*b and Haitao Huang ${ }^{\star a}$

Correction for 'Nitride MXenes as sulfur hosts for thermodynamic and kinetic suppression of polysulfide shuttling: a computational study' by Ke Fan et al., J. Mater. Chem. A, 2021, 9, 25391-25398, DOI: 10.1039/D1TA06759A.

In the original article, the position of the Gibbs free energy of $\mathrm{Li}_{2} \mathrm{~S}_{2}{ }^{*}$ on $\mathrm{V}_{2} \mathrm{NS}_{2}$ in Fig. 6 is incorrect. All the Gibbs free energy values in the original article are correct and the conclusions are not affected. The corrected Fig. 6 is shown below:

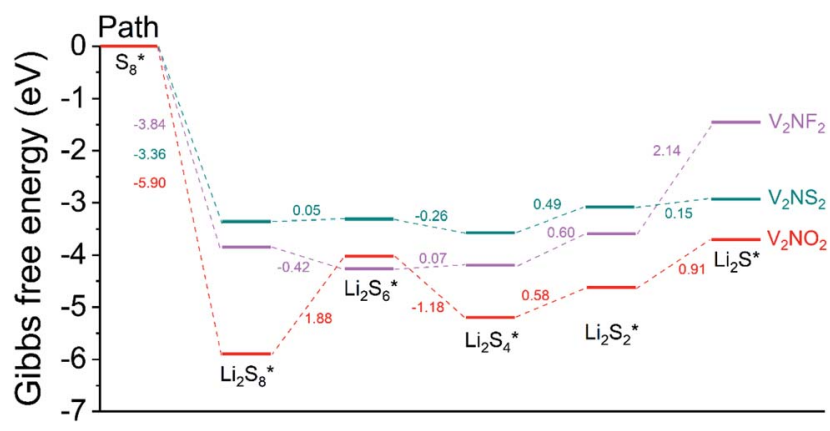

Fig. 6 Gibbs free energy profiles for the sulfur reduction reaction on $\mathrm{V}_{2} \mathrm{NO}_{2}, \mathrm{~V}_{2} \mathrm{NF}_{2}$, and $\mathrm{V}_{2} \mathrm{NS}_{2}$.

The Royal Society of Chemistry apologises for these errors and any consequent inconvenience to authors and readers.

\footnotetext{
${ }^{a}$ Department of Applied Physics, Research Institute for Smart Energy, The Hong Kong Polytechnic University, Hung Hom, Kowloon, Hong Kong, P. R. China. E-mail: aphhuang@ polyu.edu.hk

${ }^{b}$ State Key Laboratory of Optoelectronic Materials and Technologies, Centre for Physical Mechanics and Biophysics, School of Physics, Sun Yat-sen University, Guangzhou, Guangdong Province, P. R. China. E-mail: luox77@mail.sysu.edu.cn
} 